\title{
Positive prostate biopsy following radiotherapy can predict metastasis-free survival in localized prostate cancer
}

\author{
A. Zapatero ${ }^{\mathrm{a}, *}$, M. Adrados ${ }^{\mathrm{b}}$, L. Torres ${ }^{\mathrm{a}}$, M.S. Talaya ${ }^{\mathrm{a}}$, A. Cruz Conde ${ }^{\mathrm{a}}$, \\ C. Martin de Vidales ${ }^{\mathrm{a}}$, L. Vega Piris ${ }^{\mathrm{c}}$, C. Olivier $^{\mathrm{d}}$, M.T. Murillo ${ }^{\mathrm{a}}$ \\ a Department of Radiation Oncology, Hospital Universitario de la Princesa, Madrid, Spain \\ b Department of Pathology, Hospital Universitario de la Princesa, Madrid, Spain \\ c Methodologic Unit Health Research Institute, Hospital Universitario de la Princesa, Madrid, Spain \\ d Department of Urology, Hospital Universitario de la Princesa, Madrid, Spain
}

\section{A R T I C L E I N F O}

\section{Article history:}

Received 28 May 2019

Received in revised form 3 September 2019

Accepted 4 December 2019

Available online 9 December 2019

\section{Keywords:}

Prostate cancer

Biopsy

Post-treatment

Radiation therapy

Intensity-modulated radiotherapy

Survival

\begin{abstract}
A B S T R A C T
Background/aim(s): To determine the impact of post-treatment biopsy results on 10-year metastasis-free survival (MFS), overall survival (OS) and cause-specific survival (CSS) in localized prostate cancer (PCa) patients treated with high-dose radiotherapy (RT).

Materials/Methods: Retrospective analysis of 232 patients with T1c-T3bN0M0 PCa who underwent a prostate biopsy 24-36 months after high-dose RT. Biopsies were categorized as positive biopsy (PB) if H\&E staining showed evidence of residual malignancy and negative biopsy (NB) if no malignant cells were present. Kaplan-Meier estimates of 10-year MFS, OS and CSS rates were calculated for each group and Cox proportional-hazards models were used to estimate the hazard ratios. The median follow-up was 124 months (range 26-267).

Results: Sixty-two of 232 (26.7\%) patients had post-treatment positive biopsies (PB). A positive posttreatment biopsy was significantly associated with a lower 10 -year MFS (78.4\% vs. 95.4\%, p=0.001, HR: 3.9, 95\% CI: 1.8-8.3). Although patients with PB had worse outcomes that those with NB, we could not show a statistically significant difference in OS ( $81.0 \%$ vs. $87.9 \%, \mathrm{p}=0.282$, HR: $1.3,95 \% \mathrm{CI}: 0.7-2.3)$ or CSS (96.2\% vs. 99.4\% ( $\mathrm{p}=0.201$, HR. 2.4, 95\% CI: 0.6-9.7). After multivariate analysis, the strongest predictor of MFS was the post-treatment biopsy status ( $<<0.001$, HR: 5.4, 95\% CI 2.26-12.85) followed by Gleason score ( $\mathrm{p}=0.002$, HR: 2.24, 95\% CI 1.33-3.79).

Conclusion: A positive biopsy following RT can predict MFS in localized prostate cancer. These data highlight the relevance of achieving a local control and support the use of aggressive local therapeutic interventions for PCa.
\end{abstract}

(c) 2019 Greater Poland Cancer Centre. Published by Elsevier B.V. All rights reserved.

\section{Background}

Post-treatment prostate biopsies provide an accurate assessment of the local efficacy of new modalities of radiation therapy (RT), such as dose escalation protocols, extreme hypofractionation schemes or combination treatments involving brachytherapy. Furthermore, biopsy remains the only direct assessment of local tumour failure in those selected patients with rising PSA that are suitable for local salvage treatment.

\footnotetext{
* Corresponding author at: Radiation Oncology Department, Hospital Universitario de la Princesa, Health Research Institute IIS-IP, Diego de León 62, 28006 Madrid, Spain.

E-mail address: almudena.zapatero@salud.madrid.org (A. Zapatero).
}

Whereas prostate biopsy positivity after RT has been consistently shown to be a significant determinant of eventual biochemical relapse, its relationship with the risk of distant metastasis and cancer specific mortality has been less thoroughly examined in the era of high-dose RT..$^{1-5}$ In this study we update 10-year results of a cohort of prostate cancer (PCa) patients who underwent prostate biopsies following high-dose RT. ${ }^{3}$

\section{Aim}

The purpose of the present analysis was to determine the impact of post-treatment biopsy findings on long-term metastasis-free survival (MFS), overall survival (OS) and cause-specific survival (CSS). 


\section{Material and methods}

As part of 2 sequential studies on prostate biomarkers, ${ }^{3,6}$ 232 patients with clinical stage T1c-T3bN0M0 PCa treated with high dose-RT underwent a planned transrectal ultrasound (TRUS)guided prostate biopsy 24-36 months after RT. Post-RT biopsy procedures and histologic assessment have been previously described. $^{3}$ In brief, sextant or greater right and left systematic biopsies were performed by the same urology team. All biopsies were cut at six levels, of which three were stained with Haematoxylin and Eosin (H\&E) and were examined by a dedicated genitourinary pathologist. Using strict criteria for pathological assessment and for purposes of the analysis, biopsies were categorized as positive biopsy (PB) if H\&E staining showed evidence of residual malignancy regardless of the degree of therapy effect identified, and negative biopsy (NB) if no malignant cells were present. In 2004, the immunohistochemical analysis with prostatic intraepithelial neoplasia (PIN) p63/amethylacyl- coenzyme-A racemase (AMACR) to demonstrate basal cells was introduced in the routine practice and was performed to distinguish radiation from residual malignancy. All biopsies displayed radiation effects.

RT technique, planning and delivery have been depicted in detail elsewhere. ${ }^{6}$ Briefly, from 1995 to 2007 PC patients were treated with the 3DCRT technique. IMRT/IGRT with the implantation of intraprostatic gold seed fiducial markers was implemented in 2007 to escalate dose from 76.0 to $80.0 \mathrm{~Gy}$ in $38-40$ fractions. The median ICRU (International Commission on Radiation Units and Measurements) radiation dose was 77.0 Gy (66.0-84.0 Gy).

One hundred and forty-three (6) patients were treated with long-term androgen deprivation (LTAD) (neoadjuvant and concomitant ADT followed by 2 years of adjuvant ADT after RT), 41 (17.7\%) patients received short-term androgen deprivation (STAD) (4-6 months of neoadjuvant and concomitant androgen deprivation), and 47 (20.3\%) patients received RT alone.

\subsection{Follow-up}

All patients were followed continuously from treatment completion. Follow-up visits, including DRE, PSA and serum testosterone determination, and assessment of specific morbidity, occurred first at 3 months for 2 years, then every 6 months for 5 years and annually thereafter. Patients in the high-risk group treated with LTAD also underwent more extensive evaluation, including CT scan, bone scan, and chest X-ray at the end of hormonal therapy as part of the multicenter study. Prostate biopsies were planned at 24-36 months after completion of RT or at the end of LTAD.

\subsection{Statistical analysis}

The baseline characteristics of the PB versus NB patients were compared using the chi-square or Fisher's exact tests for categorical data and the Student's t or Mann-Whitney tests for continuous data. PSA relapse was defined according to the Phoenix consensus definition as an absolute nadir PSA plus $2 \mathrm{ng} / \mathrm{ml}$. Probabilities for OS, CSS and MFS were calculated using Kaplan-Meier product limited estimates from the date of diagnosis of PCa to the last follow-up or death and differences were tested by the log-rank test. Patients dead due to unrelated causes were censored at death. CSS was calculated from date of diagnosis till death due to PCa. MFS was defined as the probability of remaining free from distant metastasis.

A univariate analysis was used to detect potential risk factors affecting outcome. Variables included in the analyses were: patient age, T stage, Gleason score, pre-treatment PSA, number of positive cores at diagnosis, radiation dose, administration and length of hormonal therapy, PSA nadir and post-treatment biopsy status.
Table 1

Baseline patient, tumor and treatment characteristics for global series $(\mathrm{N}=232)$ and for the subgroup of patients with re-biopsy under biochemical control conditions $(\mathrm{N}=168)$

\begin{tabular}{|c|c|c|c|}
\hline & & $\mathrm{N}=232$ & $N=168^{a}$ \\
\hline \multicolumn{2}{|c|}{ Median follow-up, months (range) } & $124(26-267)$ & $123(42-267)$ \\
\hline \multicolumn{2}{|c|}{ Median age, years (range) } & $68(47-78)$ & $68(51-78)$ \\
\hline \multirow[t]{3}{*}{ Clinical T Stage } & $\mathrm{T} 1$ & $33(14 \%)$ & $19(11 \%)$ \\
\hline & $\mathrm{T} 2$ & $71(31 \%)$ & $44(26 \%)$ \\
\hline & $\mathrm{T} 3$ & $128(55 \%)$ & $105(63 \%)$ \\
\hline \multirow[t]{3}{*}{ Gleason score } & $\leq 6$ & $109(47 \%)$ & $74(44 \%)$ \\
\hline & 7 & $81(35 \%)$ & $62(37 \%)$ \\
\hline & $8-10$ & $42(18 \%)$ & $32(19 \%)$ \\
\hline \multicolumn{2}{|c|}{ Median PSA ng/ml (range) } & $16(4-420)$ & $17(4-420)$ \\
\hline & $\geq 10$ & $69(30 \%)$ & $43(26 \%)$ \\
\hline & $10-20$ & $76(33 \%)$ & $56(33 \%)$ \\
\hline & $>20$ & $87(37 \%)$ & $69(41 \%)$ \\
\hline \multirow[t]{3}{*}{ Risk groups } & Low & $19(8 \%)$ & $7(4 \%)$ \\
\hline & Intermediate & $44(19 \%)$ & $25(15 \%)$ \\
\hline & High & $169(73 \%)$ & $136(81 \%)$ \\
\hline \multicolumn{2}{|c|}{$\mathrm{N}^{\circ}(+)$ cores (median, range) } & $3(1-8)$ & $3(1-8)$ \\
\hline \multicolumn{2}{|c|}{ Median RT dose, Gy (range) } & $77(60-84)$ & $78(60-83)$ \\
\hline \multirow{5}{*}{ Hormones } & $\leq 78$ & $128(55 \%)$ & $84(50 \%)$ \\
\hline & $>78$ & $103(45 \%)$ & $83(50 \%)$ \\
\hline & No & $47(20 \%)$ & $18(11 \%)$ \\
\hline & STAD & $41(18 \%)$ & $23(14 \%)$ \\
\hline & LTAD & $143(62 \%)$ & $126(75 \%)$ \\
\hline
\end{tabular}

RT: Radiotherapy; STAD: Short-term androgen deprivation; LTAD: long-term androgen deprivation.

a Subgroup of patients with re-biopsy under biochemical control conditions.

Variables with a statistical significance $<0.15$ in the univariate analysis and those considered to be of clinical relevance were included in the multivariate model. A p $<0.05$ significance level (2-sided) was considered for all statistical tests. Statistical analysis was performed using SPSS version 10.0 (SPSS, Chicago, IL).

\section{Results}

The median follow-up was 124 months (range 26-267). One hundred and sixty-nine (73\%) patients had high-risk PCa according to NCCN definition. Median RT dose was 77 Gy. LTAD was administered in 143 (62\%) patients. Patients and treatment characteristics are summarized in Table 1.

Sixty-two (26.7\%) patients presented histological failure or PB following treatment. Of the variables analysed, only the PSA nadir $(p=0.019)$ and the use of hormone therapy $(p<0.001)$ were significantly associated with a higher histological failure rate, while patient age, clinical T stage, Gleason sum, pre-treatment PSA, number of positive cores at diagnosis, risk group and radiation dose were not (Table 2).

\subsection{Outcome data}

Overall, 178/232 (76.7\%) patients are currently alive, 28 (12.1\%) patients developed distant metastasis and $8(3.4 \%)$ patients died of PCA. Patients with a positive re-biopsy were more likely to develop a biochemical failure in the future. The 10-year biochemical disease-free survival (bDFS) in patients with NB was 69.9\% compared to $20.6 \%$ in patients with PB results $(\mathrm{p}<0.001$, HR 5.12 95\% CI 3.41-7.68). The overall 10-year OS, CSS and MFS were respectively $86.0 \%, 97.5 \%$ and $90.7 \%$. Although patients with $\mathrm{PB}$ had worse outcomes that those with NB, we could not show a statistically significant difference in OS (PB: $81.0 \%$ vs. NB: $87.9 \%$, $\mathrm{p}=0.282$, HR: $1.3,95 \% \mathrm{CI}: 0.7-2.3$ ) or CSS (PB: $92.6 \%$ vs. NB: $99.4 \%$ ( $p=0.201$, HR. 2.4, 95\% CI: 0.6-9.7). However, we found that the post-treatment biopsy status was significantly associated with the MFS. The 10-year MFS was $78.4 \%$ for PB vs. $95.4 \%$ for NB, $\mathrm{p}<0.001$, HR: 3.9, 95\% CI: 1.8-8.3 (Fig. 1). 
Table 2

Correlations of post-RT biopsy results with clinical and treatment factors.

\begin{tabular}{|c|c|c|c|c|}
\hline & PB 62 & NB 170 & P value & \\
\hline \multirow[t]{2}{*}{ Patient age (years) } & $\leq 65$ & 27 & 59 & 0.217 \\
\hline & $<65$ & 35 & 111 & \\
\hline \multirow[t]{3}{*}{ Clinical T Stage } & $\mathrm{T} 1$ & 13 & 20 & 0.204 \\
\hline & $\mathrm{T} 2$ & 18 & 53 & \\
\hline & T3 & 31 & 97 & \\
\hline \multirow[t]{3}{*}{ Gleason score } & $\leq 6$ & 31 & 78 & 0.843 \\
\hline & $\overline{7}$ & 20 & 61 & \\
\hline & $8-10$ & 11 & 31 & \\
\hline \multirow[t]{3}{*}{ Pretreament PSA } & $\geq 10 \mathrm{ng} / \mathrm{ml}$ & 22 & 47 & 0.509 \\
\hline & $10-20$ & 19 & 57 & \\
\hline & $>20$ & 21 & 66 & \\
\hline \multirow[t]{3}{*}{ Risk groups } & Low & 8 & 11 & 0.097 \\
\hline & Intermediate & 15 & 25 & \\
\hline & High & 39 & 130 & \\
\hline \multirow[t]{2}{*}{$\mathrm{N}^{\mathrm{o}}(+)$ cores at diagnosis } & $1-3$ & 27 & 100 & 0.090 \\
\hline & $>3$ & 28 & 61 & \\
\hline \multirow[t]{2}{*}{ RT dose } & $\leq 78 \mathrm{~Gy}$ & 35 & 93 & 0.719 \\
\hline & $>78 \mathrm{~Gy}$ & 26 & 77 & \\
\hline \multirow[t]{3}{*}{ Hormones } & No & 23 & 24 & $<0.001$ \\
\hline & STAD & 13 & 28 & \\
\hline & LTAD & 25 & 118 & \\
\hline \multirow[t]{3}{*}{ PSA nadir } & $\leq 1 \mathrm{ng} / \mathrm{ml}$ & 51 & 160 & 0.019 \\
\hline & $1-2 \mathrm{ng} / \mathrm{ml}$ & 8 & 8 & \\
\hline & $>2 \mathrm{ng} / \mathrm{ml}$ & 3 & 2 & \\
\hline
\end{tabular}

PB: Positive biopsy; NB: Negative biopsy; RT: Radiation therapy; STAD: Short-term androgen deprivation; LTAD: long-term androgen deprivation.

Table 3

Summary of univariate and multivariate analysis of clinical and treatment factors of potential factors affecting metastasis-free survival $(\mathrm{N}=232)$.

\begin{tabular}{llllll}
\hline & \multicolumn{2}{l}{ Univariate } & & \multicolumn{2}{l}{ Multivariate } \\
\cline { 2 - 3 } VARIABLES & p value & HR $($ IC95\%) & & p value & HR (IC95\%) \\
\hline Patient age & 0.448 & $1.33(0.63-2.81)$ & & \\
T stage (T3 vs T1-2) & 0.126 & $1.83(0.82-4.10)$ & & \\
Gleason sum (>7) & $<0.001$ & $5.64(2.33-13.63)$ & 0.002 & $2.24(1.33-3.79)$ \\
Pretreatment PSA $(>20)$ & 0.582 & $1.63(0.61-14.30)$ & & \\
Risk group (high risk) & 0.249 & $3.33(0.44-24.78)$ & & \\
$\mathrm{N}^{0}(+)$ cores (>3) & 0.002 & $3.86(1.58-9.40)$ & & \\
Radiation dose $(>78$ Gy) & 0.512 & $0.76(0.24-1.72)$ & & \\
Hormone therapy & 0.393 & $1.82(0.76-4.37)$ & & \\
PSA nadir & 0.051 & $1.94(0.99-380)$ & & \\
Positive biopsies & $<0.001$ & $3.90(1.82-8.37)$ & $<0.001$ & $5.4(2.26-12.85)$ \\
\hline
\end{tabular}

HR: Hazard ratio; $\mathrm{CI}$ : Confidence interval.

\subsection{Risk factors analysis for metastasis free survival}

The results of univariate analysis showed that Gleason sum $(p<0.001)$, the number of positive cores at diagnosis $(p=0.002)$ and post-treatment biopsy results $(\mathrm{p}<0.001)$ were significantly associated with MFS. The MVA demonstrated that the strongest predictor of MFS was the post-treatment biopsy status ( $\mathrm{p}<0.001$, HR: $5.4,95 \%$ CI 2.26-12.85) followed by Gleason score ( $p=0.002$, HR: $2.24,95 \%$ CI 1.33-3.79) (Table 3).

\subsection{Subgroup analysis}

Of the 232 patients, $168(72.4 \%)$ had biochemical and clinical control at the time of the post-RT biopsy. Twenty-five of the 168 (14.8\%) patients presented histological failure or PB following treatment. We performed a separate analysis in this specific subgroup in order to verify the impact of $\mathrm{PB}$ on long-term outcomes under biochemical and clinical control conditions. One hundred and thirty-six (81\%) patients in this subgroup had high risk PCa and received more intensive treatment (median RT dose was $78.0 \mathrm{~Gy}$, and LTAD was administered in 126 (75\%) patients. The overall 10-
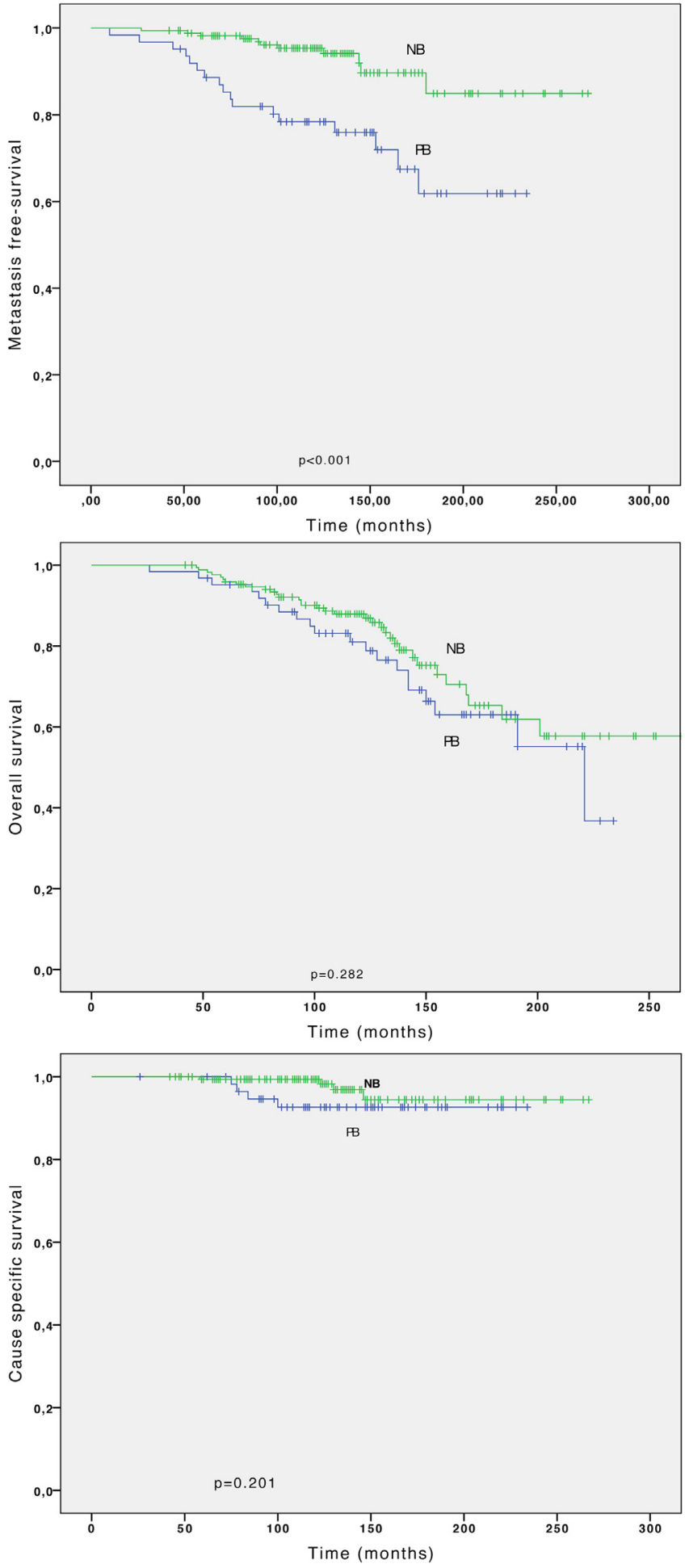

Fig. 1. Cumulative probability of metastasis-free survival (MFS), overall survival (OS) and cause-specific survival (CSS) according to post-treatment biopsy status (PB: positive biopsy; NB: negative biopsy) for the whole series $(\mathrm{N}=232)$.

year probability of OS, CSS and MFS were $89.2 \%, 1.0 \%$ and $94 \%$, respectively.

Similar to the whole series, we also found in this subgroup of patients that the post-treatment biopsy status was significantly associated with the MFS. The 10 -year MFS was $78.9 \%$ for patients with a PB vs. $96.7 \%$ for those with a NB, p<0.001, HR: $7.095 \% \mathrm{CI}$ : 2.2-22.4) (Fig. 2). The results of univariate and MVA confirmed that the post-treatment biopsy status $(p=0.001$, HR: $8.63,95 \%$ 

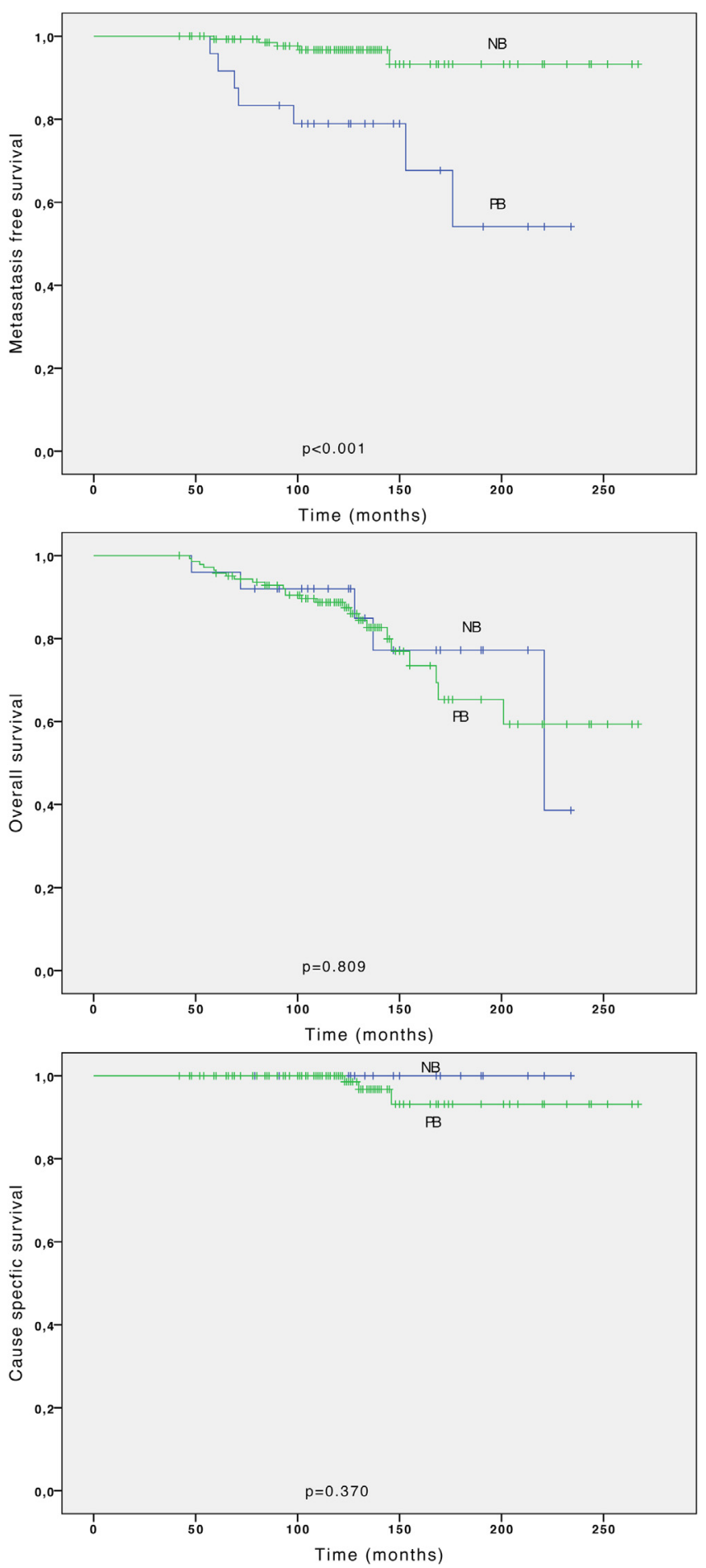

Fig. 2. Cumulative probability of metastasis-free survival (MFS), overall survival (OS) and cause-specific survival (CSS) according to post-treatment biopsy status (PB: positive biopsy; NB: negative biopsy) for the subgroup of patients under biochemical and clinical conditions $(\mathrm{N}=168)$.

CI 2.31-32-26) and Gleason score ( $p=0.041$, HR: $6.03,95 \% \mathrm{CI}$ 1.07-33.58) were independent factors affecting the risk of MFS.

\section{Discussion}

In the present study, a PB following RT was associated with a fivefold increased risk of distant metastases. The 10-year MFS was $78.4 \%$ for patients with a PB vs. $95.4 \%$ for those with a NB $(p<0.001)$.
This relationship also confirmed in the subgroup of patients with PSA and clinical control at re-biopsy, highlights the relevance of achieving a local control, supports the use of aggressive local therapeutic interventions and confirm that a failure to control the local disease within the prostate predisposes patients to developing distant metastases also in the unfavorable group of high-risk locally advanced PCa.

The relationship between the disease-free survival outcome and biopsy status has been reported previously. 1,4,7,8 More recently, long-term data of post-treatment biopsies in cohorts of patients participating in randomized trials have also confirmed these findings. ${ }^{9}$ However, the majority of these studies were largely focused on the association with biochemical failure.

The direct impact of a PB after RT on the risk of distant metastasis has been less thoroughly studied and with controversial results. Coen et al. found that local failure was the strongest predictor for distant metastases in a multivariate model. ${ }^{5}$ The main limitation was that the definition of local failure used by the authors included either palpable recurrence or positive re-biopsy, or malignant cells identified on a subsequent transurethral resection of the prostate. In fact, a positive re-biopsy was described only in 60 of the 198 patients with local failure. Similarly, although Pollack et al. observed a significant relationship between the 5-year freedom from relapse (which included biochemical and/or disease failure) and the post-treatment biopsy results $(\mathrm{p}=0.0002)$, no direct impact on MFS was reported. ${ }^{8}$ More interestingly, Solberg et al. ${ }^{2}$ in a sub-study of 120 patients from the SPCG7 trial of locally advanced PCa treated with ADT with or without 70 Gy RT, found that residual prostate cancer (in re-biopsies at 45 months post treatment) was significantly associated with serum prostate-specific antigen recurrence, local tumour progression, clinical recurrence, and cancer-specific death in univariable analysis, but failed to show a significant correlation with distant metastasis $(\mathrm{p}=0.27)$.

Conversely, there have been a couple of studies that have assessed and reported a significant relationship between metastasis-free survival and a positive re-biopsy. Zelefsky et al., in a series of 339 patients with post-RT biopsy and a median FU of 10 years, showed an inferior distant metastasis-free survival in patients with a positive post-RT biopsy. The 10-year MFS rate was $69 \%$ in patients with a PB compared to $90 \%(\mathrm{p}=0.0004)$ in patients with a NB. ${ }^{10}$ More recently, Krauss et al. ${ }^{11}$ reported the longest series of re-biopsy post RT. The authors performed a retrospective analysis of a subgroup of 831 low risk patients from the RTOG 9408 trial. With a median follow-up of 9 years they demonstrated that a post-treatment PB was significantly associated with increased rates of DM and inferior DSS in patients treated with definitive RT. The 10-year estimate of distant metastases (DM) was $8 \%$ vs. $4 \%$, [HR $=2.4 ; 95 \%$ CI 1.3-4.4]. Of note, this series did not include patients with locally advanced or high-risk tumors, the RT dose was suboptimal for the current standards and the hormone therapy used in the investigation arm was continued for 4 months.

Our series with a median follow-up of 10 years confirms the relationship between a post-RT PB and a higher risk of distant metastasis. The 10 -year MFS results (95.4\% vs. $78.4 \%, p=0.001$, HR: $3.9,95 \% \mathrm{CI}: 1.8-8.3$ ) are in agreement with the above mentioned data. What makes our study distinctive from prior reports is that $81 \%$ of the patients had high-risk disease and were treated with modern intensive treatment (median radiation dose $78 \mathrm{~Gy}$ and LTAD in 75\%), and that the re-biopsy was performed under biochemical and clinical control conditions. Present data regarding the significant impact on bDFS confirm our previous report. ${ }^{3}$ However, although we observed that the patients with a post-RT PB had a worse OS and CSS than those with a NB, in this report we failed to show a statistically significant value. The relatively small sample size of the study and the low number of events could be responsible for the lack of a significant impact. Recently, a systematic review 
of data from randomized trials on localized PCa acknowledged the value of MFS as a valid and strong surrogate for OS for localized PCa. ${ }^{12}$ Of note, the use of MFS can allow an expeditious evaluation of a new therapy if it has a meaningful treatment effect on MFS.

The positive biopsy rate of our study was $26.7 \%$ in the whole series and only $14.8 \%$ in the group without PSA failure. These results are challenging since $81 \%$ of patients had high-risk disease and could be explained by the use of more intensive treatment. Unlike the results of other authors, ${ }^{10,11}$ we did not find a significant association between known clinical, pathologic and treatment factors and a higher detection of PB. Again, the small sample size and number of events could explain these findings. Only the number of positive cores at diagnosis was found to be associated with the re-biopsy status.

Although multiparametric resonance imaging (mpMRI) of the prostate has become a reliable method to diagnose local tumour extension, its value to detect local recurrence after RT has been less extensively explored. ${ }^{13}$ Most of these studies have small sample sizes and lack histological proof of tumor location. One recent series analyzed the diagnostic performance of mpMRI in 19 patients with biopsy-proven recurrent PCa after primary RT that underwent salvage prostatectomy. ${ }^{14}$ The results showed a $50-71 \%$ sensitivity with $80-100 \%$ specificity for detecting extra-prostatic extension. Other studies of mpMRI validated by transperineal systematic template prostate mapping (TPM) have shown a good performance for detection of radio-recurrent prostate cancer with an area under the curve of $0.84 .{ }^{15,16}$ All these studies are promising and exciting, however, until a full validation of functional imaging as a potential surrogate is made, post-treatment biopsy remains the only reliable procedure for a direct assessment of tumor control in the evaluation of new modalities of local therapies, and of selected patients with rising PSA that are suitable for local salvage treatment.

Notwithstanding the significant association between a post-RT positive biopsy and MFS, prostate re-biopsy is not recommended routinely in clinical practice since it is an invasive procedure with the risk of infection and bleeding. Furthermore, we cannot obviate that the timing of post-treatment biopsies is challenging with false-positive results. Finally, pathology interpretation is not straightforward and requires an experimented uropathologist.

The authors recognize the limitations inherent to the retrospective evaluation of the study. Besides, the sample size was relatively limited and the number of survival events was low. The strength and distinction of the present study is the long-follow up (median 123 months), the presence of $81 \%$ of patients with high-risk PCa that were treated with modern intensive treatment, and the re-biopsy performed under biochemical and clinical control conditions.

\section{Conclusion}

The long-term results of the present study indicate that a positive biopsy following RT portends inferior MFS in prostate cancer. These data highlight the critical importance of a successful local therapy in men with high-risk prostate cancer and support the role of post-treatment biopsy in the validation of new therapies.

\section{Conflict of interest}

I certify that: None of the contributing authors (including myself) have any conflicts of interest including any financial sup- port, any grants and any financial interests or activities or any relationships or affiliations relevant to the subject matter or materials discussed in the manuscript.

\section{Financial disclosure}

I certify that: None of the contributing authors have any conflicts of interest including specific financial interests and relationships and affiliations relevant to the subject matter or materials discussed in the manuscript.

\section{References}

1. Vance Waseet, Tucker Susan L, Crevoisier Renaud De, Kuban Deborah A, Rex Cheung M. The predictive value of 2-year posttreatment biopsy after prostate cancer radiotherapy for eventual biochemical outcome. Int J Radiat Oncol Biol Phys. 2007;67:828-833.

2. Solberg Arne, Haugen Olav A, Yz Trond Viset, et al. Residual prostate cancer in patients treated with endocrine therapy with or without radical radiotherapy: A side study of the SPCG-7 randomized trial. Int J Radiat Oncol Biol Phys. 2011:80:55-61.

3. Zapatero Almudena, Mínguez Ricardo, Nieto Santiago, Martín de Vidales Carmen, García-Vicente Feliciano. Post-Treatment Prostate Biopsies In The Era Of Three-Dimensional Conformal Radiotherapy: What Can They Teach Us? Eur Urol. 2009:55:902-910.

4. Crook J, Malone S, Perry G, Bahadur Yasir, Robertson Susan, Abdolell Mohamed. Postradiotherapy prostate biopsies: What do they really mean? Results for 498 patients. Int J Radiat Oncol Biol Phys. 2000;48:355-367.

5. Coen John J, Zietman Anthony L, Thakral Harjot, Shipley William U. Radical radiation for localized prostate cancer: Local persistence of disease results in a late wave of metastases. J Clin Oncol. 2002;20:3199-3205.

6. Zapatero Almudena, Morente Manuel, Nieto Santiago, Martín de Vidales Carmen, Lopez Consuelo, Adrados Magdalena, et al. Predictive Value of PAK6 and PSMB4 Expression In Patients with Localized Prostate Cancer Treated with Dose-Escalation Radiation Therapy and Androgen Deprivation Therapy. Urologic Oncology: Seminars and original investigations. 2014:32:1327-1332.

7. D’Alimonte Laura, Helou Joelle, Sherman Christopher, et al. The clinical significance of persistent cancer cells on prostate biopsy after high-dose-rate brachytherapy boost for intermediate-risk prostate cancer. Brachytherapy. 2015;14:309-314.

8. Pollack Alan, Zagars GunarK, Antolak John A, Kuban Deborah A, Rosen Isaac I. Prostate biopsy status and PSA nadir level as early surrogates for treatment failure: Analysis of a prostate cancer randomized radiation dose escalation trial. Int J Radiat Oncol Biol Phys. 2002;54:677-685.

9. Antoine Kass-Iliyya AB, Gordana Jovic A, Claire Murphy A, et al. Twoyears postradiotherapy biopsies: Lessons from MRC RT01 trial. Eurol Urol. 2018;73:968-976

10. Zelefsky Michael J, Reuter Victor E, Fuks Zvi, Scardino Peter, Shippy Alison. Influence of local tumor control on distant metastases and cancer related mortality after external beam radiotherapy for prostate Cancer. J Urol. 2008;179(4):1368-1373

11. Krauss DJ, Hu C, Bahary JP, et al. Importance of local control in early stage prostate cancer: Outcomes of patients with positive post-radiation therapy biopsy results treated in RTOG 9408. Int J Radiat Oncol Biol Phys. 2015;92:863-873.

12. Xie Wanling, Regan Meredith M, Buyse Marc, et al. On behalf of the ICECaP Working Group. Metastasis-free survival is a strong surrogate of overall survival in localized prostate cancer. J Clin Oncol. 2017;35:3097-3104.

13. Arrayeh Elnasif, Westphalen Antonio C, Kurhanewicz John, et al. does local recurrence of prostate cancer after radiation therapy occur at the site of primary tumor? Results of a longitudinal MRI and MRSI study. Int J Radiat Oncol Biol Phys. 2012;82(5):E787-E793

14. Zattoni F, Kawashima A, Morlacco A, et al. Detection of recurrent prostate cancer after primary radiation therapy: An evaluation of the role of multiparametric 3T magnetic resonance imaging with endorectal coil. Pract Radiat Oncol. 2017; 7:42-49.

15. Abd-Alazeez M, Ramachandran N, Dikaios N, et al Multiparametric MRI for detection of radiorecurrent prostate cancer: Added value of apparent diffusion coefficient maps and dynamic contrast-enhanced images. Prostate Cancer Prostatic Dis. 2015;18:128-136.

16. Kanthabalan A, Abd-Alazeez M, Arya M, et al. Transperineal magnetic resonance imaging-targeted biopsy versus transperineal template prostate mapping biopsy in the detection of localised radio-recurrent prostate cancer. Clin Oncol (R Coll Radiol). 2016;28(9):568-576. 